\title{
COMUNICACIÓN EMOCIONAL NO VERBAL EN LA INFANCIA, ANÁLISIS EN EL AULA DESDE LA METODOLOGÍA OBSERVACIONAL
}

\author{
Leidy Tatiana Porras Cruz, Rafael Enrique Buitrago Bonilla y Adriana Marien Gutiérrez Torrez \\ Universidad Pedagógica y Tecnológica de Colombia, Colombia
}

\section{Resumen}

El presente estudio analizó la comunicación emocional no verbal a partir de los comportamientos gestuales de 21 alumnos del grado de transición de educación infantil de la Institución Educativa Instituto Técnico Gonzalo Suárez Rendón, de carácter público en la ciudad de Tunja - Colombia. La investigación se fundamentó en la metodología observacional (Anguera, 2010; Anguera et al., 2001), e hizo uso de un instrumento no estándar (Anguera-Argilaga et al., 2007). Dentro de los resultados obtenidos se destaca la presencia de un alto porcentaje de gestos ilustradores, seguido de gestos reguladores y un porcentaje menor en los gestos adaptadores. En consecuencia, se ratificó, que la comunicación emocional no verbal acompaña la comunicación verbal y aporta a las relaciones interpersonales y sociales de las niñas y niños.

Palabras clave: Inteligencia Emocional, Educación Infantil, Comunicación.

\section{Abstract}

The present study analyzed the non-verbal emotional communication from the gestural behaviors of 21 students of early childhood education of the public Educative Institution "Instituto Técnico Gonzalo Suárez Rendón" in Tunja City, Colombia. The research was based on observational methodology (Anguera, 2010; Anguera et al., 2001), and non-standard instrument was used (Anguera-Argilaga et al., 2007). Among the results obtained, the presence of a high percentage of illustrative gestures stands out, followed by regulatory gestures, and a lower percentage of the adaptive gestures. Therefore, it was confirmed that non-verbal emotional communication accompanies verbal communication and helps the interpersonal and social relationships of girls and boys.

Keywords: Emotional Intelligence, Early Childhood Education, Communication.

Correspondencia.

Rafael Enrique Buitrago Bonilla

rafael.buitrago@uptc.edu.co

Universidad Pedagógica y Tecnológica de Colombia 


\section{Introducción}

Las expresiones faciales y corporales posibilitan la exteriorización y reconocimiento de emociones (en particular las universales: alegría, ira, miedo, tristeza, sorpresa y asco), al igual que motivaciones, intenciones, actitudes y reacciones ante distintos estímulos internos y externos. Por consiguiente, las expresiones aportan a la comprensión de las personas y facilitan la comunicación, la interacción y la generación de vínculos al integrar, como es lógico, lo verbal y lo no verbal (Deutsch, 1947; Ekman y Friesen, 1972; Mayer et al., 2016; Olderbak et al., 2019; Yadav y Singha, 2020).

En este sentido, comprender y decodificar el lenguaje no verbal de los demás, requiere el desarrollo de habilidades emocionales, debido a que estas manifestaciones corporales están cargadas de significados emocionales que son fundamentales en las relaciones interpersonales, debido a que permiten entender a la otra persona e interactuar de mejor manera con ella. En consecuencia, en el contexto escolar la identificación e interpretación del lenguaje no verbal de los estudiantes, tiene una relación estrecha con las habilidades socio emocionales del docente (Carpena y Darder, 2010; Extremera et al., 2019; Palomera et al., 2017) lo que, a su vez, favorece la mediación y gestión del clima escolar (Buitrago-Bonilla y Cárdenas-Soler, 2017).

Desde la perspectiva del estudio de las emociones, Pert (1986) señala que, los neuropéptidos son la clave bioquímica de las emociones, de la relación entre mente y cuerpo, así como de la expresión emocional, por consiguiente, se pueden considerar a las emociones como impulsos innatos $\mathrm{y}$ necesarios que, haciendo uso del sistema nervioso central, cumplen una función adaptativa y determinan las acciones humanas como las reacciones, comportamientos y el aprendizaje, aspectos determinantes en las relaciones entre las personas (Perlovsky y Schoeller, 2019).

Además de ello, en la actualidad se reconoce la intervención tanto de la amígdala como de los lóbulos frontales en los procesos emocionales, pero también, el papel protagónico de la corteza prefrontal en la regulación emocional, habilidad que es distintivamente humana (Davidson, 2003a; Linden, 2010; Ochsner et al., 2004). Lo cierto es que aspectos como el reconocimiento emocional, la regulación, la memoria emocional y los cambios de comportamiento están soportados en circuitos corticales y subcorticales, además de ello, que la amígdala es relevante en la primera fase del aprendizaje de los estilos afectivos, sin embargo, su importancia se reduce una vez éstos se han aprendido (Davidson, 2003b).

Es importante resaltar que, durante la infancia se perfecciona la capacidad de reconocer la intensidad de las emociones, aspecto fundamental para la comunicación interperso- nal y para los procesos de aprendizaje, ya que las emociones lo pueden fomentar o detener (García y Tully, 2020; Gordillo et al., 2015). Además de ello, se ha evidenciado que en las niñas y niños de preescolar el reconocimiento e interpretación de emociones como la tristeza o la angustia a través de su manifestación gestual y corporal, inciden en sus relaciones sociales y emocionales y por lo tanto en el clima de aula (Holm y Cekaite, 2021). En este sentido, diversas investigaciones han probado que los niños inician categorizando las emociones como positivas y negativas, diferenciando de una forma más puntual las emociones de disgusto y miedo, lo cual sugiere que éstas son utilizadas para comprender y empatizar con sus pares teniendo repercusiones dentro y fuera del aula (Durand et al., 2007; Fong et al., 2020; Vicari et al., 2000; Widen y Russell, 2003).

De igual manera, es importante señalar que las niñas y los niños, al igual que las demás personas, hacen uso de su lenguaje corporal para potenciar las expresiones emocionales, sugiriendo que en contextos sociales e interactivos se realiza una interpretación de la comunicación en base a las expresiones emocionales del otro, lo cual posibilita la resolución de problemas (Ip et al., 2021; Repacholi y Meltzoff, 2007; Walle et al., 2017), lo que además, como es lógico, se evidencia también en las prácticas colectivas y formativas con el reconocimiento y expresión de emociones entre infantes (Denervaud et al., 2020). Por ello, en la medida en que los niños crecen, empiezan a utilizar con más frecuencia los gestos en sus procesos comunicativos, en cualquier caso, Shapiro (2008) señala que los niños entre los 6 y 12 meses empiezan a reconocer las expresiones emocionales básicas de los cuidadores, a lo que se suma, que otros estudios han enfatizado en la comprensión emocional de algunos tipos de gesto desde los tres años (Demir-Lira et al., 2018; Goldin-Meadow, 2005; Leite, y Aureliano, 2016; Özçalışkan et al., 2016; Sekine et al., 2015).

En esta dirección, Białek et al. (2018) demostraron con un estudio longitudinal con niñas y niños, que la capacidad para comprender el significado de los gestos a los tres años era en promedio de un $46 \%$, pero que aumenta con la edad, de modo que los niños entre 4 y 6 años relacionan el $76 \%$ de los gestos con la imagen correcta, estos resultados se asocian con la comprensión y producción de los gestos en el aprendizaje de las palabras (Lavelli y Majorano, 2016; Lavelli et al., 2015; Lüke y Ritterfeld, 2014; MainelaArnold y Evans, 2014; Sánchez, 2019; Vogt y Kauschke, 2017; Weismer y Hesketh, 1993).

\section{Comunicación Emocional no Verbal}

La comunicación es un proceso complejo que permite establecer vínculos interacciones y redes entre las personas y, como es lógico, la escuela es un escenario privilegiado para ello, y si bien es cierto que en gran medida la comunicación ha sido estudiada desde las competencias lingüísticas, tam- 
bién debe ser abordada desde la perspectiva de la información no verbal, en donde se analizan aspectos sociales, culturales y geográficos del ser humano, que son utilizados como sistemas no lingüísticos para comunicarse de manera directa o para potenciar el lenguaje verbal (Cestero, 2014, 2015, 2016, 2018; Hwang y Matsumoto, 2016; Poyatos, 1994).

Así pues, la comunicación como proceso bilateral se constituye como un circuito en el que interactúan y se interrelacionan dos o más personas a través de un conjunto de signos o símbolos conocidos por los interlocutores, en donde es visible el protagonismo de las emociones. Ciertamente, se consolidan procesos cognitivos y emocionales que influyen en la toma de decisiones y acciones, en las que el cuerpo se establece como elemento comunicador desde lo no verbal, incluyendo gestos, miradas, proxemia y kinesia, siendo éstos no solo una extensión lingüística, sino elementos fundamentales en el proceso comunicativo (Bunglowala y Bonglowala, 2015; Cyrulnik, 2020; Diez y Freijeiro, 2005; Gordillo et al., 2014; Quiñones y Romero, 2020). De esta manera, la interpretación de significados emocionales en la comunicación se puede establecer como una habilidad que permite comprender los estados emocionales propios y de los demás, con la finalidad de relacionarse de forma apropiada y transmitir por medio de una correcta expresión verbal y no verbal mensajes a los demás, lo que facilita la empatía y la proximidad entre las personas (Carpena y Darder, 2010; Goleman, 2012).

En las niñas y niños esta interacción emocional es fundamental pues resalta el valor de los lazos afectivos, los cuales constituyen un canal a través del cual el infante trasmite sus necesidades de una forma implícita en la comunicación interpersonal. Estos aspectos se han evidenciado en varias investigaciones que determinan cómo las posturas corporales y los gestos, permiten el reconocimiento del estado afectivo de las niñas y niños en el aula infantil, una tarea difícil en el ámbito educativo (De Santiago, 2005; Del Barrio y Borragán, 2005, D’ Mello et al., 2007; Etchevers, 2006; Holm y Cekaite, 2021). Para tal fin, se deben tener en cuenta los sistemas de comunicación no verbal en la infancia, para poder establecer desde las huellas del comportamiento, las acciones concretas con objetos, la utilización del movimiento, la postura, la gestualidad, la mirada y las expresiones faciales de los niños, las cuales influyen de manera emocional en las relaciones escolares, razón por la que se hace necesario posicionar la comunicación no verbal y su comprensión en la escuela y el proceso educativo (Barrett et al., 2019; Matsumoto et al, 2012; McNeill, 2016; Ricci y Cortessi, 1980; Quiñones y Romero, 2020).

Respecto a la clasificación del comportamiento no verbal, Jiménez (1981) lo agrupa en cuatro grandes categorías: la kinesia, la proxemia, la paralingüística y los aspectos olfati- vos y cutáneos en la interacción social. En la presente investigación se escogió como eje de análisis la kinesia, dada su importancia en la comunicación escolar, pues, se define como la disciplina que estudia el significado de los movimientos y gestos humanos que integran las posturas corporales, las expresiones faciales y los comportamientos gestuales, expresados en códigos no verbales que en su totalidad se ven afectados por la cultura y formas de vida. Esta información, que tiene raíz emocional, puede ser transmitida por las actitudes y pensamientos entre los individuos mediante la comunicación no verbal y las expresiones faciales que evidencian el estado emocional de la persona. En los infantes, estas manifestaciones no verbales suelen ser muy evidentes dadas sus interacciones sociales reales, en donde la emocionalidad está implícita todo el tiempo (Albaladejo, 2007; Burgoon et al., 2016; Eco y Volli, 1970; Ekman y Friesen, 1969; Ip et al., 2021; Quiñones y RomerR, 2020; Yadav y Singha, 2020).

\section{Comportamientos Gestuales}

Desde la infancia, se configuran los procesos de comunicación no verbal mediante la incorporación de expresiones y gestos aprendidos en el contexto sociocultural, los cuales establecen una relación del cuerpo en inter-acción con la cultura, por ello, al hablar de cuerpo y corporeidad se habla de vida, movimiento, expresión, coexistencia y comunicación, acciones que están presentes en la escuela (Buriticá y Saldarriaga, 2020; Martí, 2017; Pozo, 2017; Tartas, 2017; Tur-Porres y Ires-Correa, 2020).

Por este motivo, es preciso especificar que en la década de 1970 el tema del gesto adquiere interés por parte de las ciencias sociales y disciplinas como la antropología, la lingüística y la psicología, en gran medida, porque retoman la concepción de cuerpo como esencia de los sistemas de comunicación no verbal, en donde los gestos se asumen como la imagen intrínseca del lenguaje, es decir, desde la perspectiva del comportamiento no-verbal en la comunicación (Durand et al., 2020; Kendon, 2004, McNeill, 2016). De manera posterior, se establecieron categorías para los gestos, teniendo en cuenta que éstos son muy personales y que responden al lenguaje como entramado lingüístico, social y artístico. En este sentido, es necesario que, en los diferentes escenarios de interacción social como la escuela, se entienda y valore el ámbito físico y comportamental de los niños, permitiendo la concepción creativa del cuerpo simbólico y comunicador (Albaladejo, 2007; Denervaud et al., 2020; Quiñones y Romero, 2020).

Es así que, para abordar y conceptualizar los gestos como movimientos psicomusculares fundamentales en el proceso de comunicación, es importante aclarar que, éstos se encuentran clasificados en dos tipos: faciales y corporales, según su función, como lo es, abrir y cerrar los canales de comunicación, y perfeccionar las respuestas verbales y no 
verbales. Acciones comprendidas en la interacción kinésica al analizar el valor de los gestos y las posturas con valor significativo y comunicativo para enviar mensajes, ya que éstos comunican el interior de cada persona, complementan el lenguaje verbal, signos convencionales y signos no lingüísticos, es decir, movimientos que expresan la personalidad y las emociones (Argyle, 1975; Fossa, 2019; Gordillo, et al., 2014). En esta misma dirección, es importante definir la postura corporal como canal de comunicación con igual importancia que la facial para transmitir emociones (Meeren et al., 2005; Van den Stock et al., 2007).

Los gestos se integran dentro de un proceso de relación social, en el cual, el empleo de los signos no verbales hacen parte de la interacción, las relaciones y los vínculos con los demás, la organización y en general con el control de la comunicación, la cual está cargada de un alto valor sociocultural que agrega información al mensaje transmitido (Alibali y Kita, 2010; Cestero, 2015, Curioni et al., 2020; Fossa, 2019; Goldin-Meadow, 2005; Özçalışkan et al, 2016; Waters y Beck, 2015). Ahora bien, los gestos se pueden clasificar en: emblemas, ilustradores, reguladores, adaptadores y señales de afecto (Knapp y Hall, 2009), en este sentido, estos fueron los aspectos abordados en el presente estudio.

Los gestos emblemas están referidos a comportamientos no verbales que pueden ser traducidos directamente a palabras y tienen un significado específico, por ejemplo, agitar la mano en acciones como el saludo o la despedida, además, se utilizan de manera consciente e intencional y, están mediados por el entorno y los lenguajes, sustituyendo palabras o frases (Belío-Apaolaza, 2019). Al respecto, Cestero (2017) propone que la integración de los signos no verbales como los emblemas se apropian de los sencillos a los complejos (Albadejo, 2007; Murillo et al., 2015), por ello, el proceso de aprendizaje de los gestos emblemáticos, en primer lugar, permite reconocer el gesto y asociarlo a su significado, en segunda instancia, comprenderlo e interpretarlo $\mathrm{y}$, en tercer lugar, producirlo adecuadamente con conciencia (Belío-Apaolaza, 2018, 2019).

Los gestos ilustradores, acompañan a la comunicación verbal y exponen el contenido del mensaje o su entonación, en otras palabras, acentúan y complementan el mensaje verbal. Son gestos batuta que cambian con los saltos de frase o de tema. Así, estos gestos tienen dos funciones, una psicológica de expresión de representaciones mentales y otra comunicacional, por lo tanto, funcionan como un sistema de puntuación que facilita la interacción entre el hablante y el oyente. Éstas expresiones no verbales, se aprenden a muy temprana edad durante la adquisición del lenguaje y resta carga cognitiva mientras la persona está pensando qué decir, en consecuencia, estos gestos se configuran como uno de los primeros medios de comunicación intencional para referirse a un entorno inmediato (Albadejo, 2007; Efron, 1941; Ekman y Friesen, 1972; Goldin-Meadow et al., 2001; Liszkowski, 2008, Lüke et al., 2017; MorenoNunez et al., 2020; Salo et al., 2019; Shablico, 2012).

Por su parte, los gestos reguladores son movimientos que intervienen en la sincronización de las intervenciones en el diálogo y mantienen el flujo de la conversación, dando indicaciones de interés. Por lo tanto, controlan de manera directa la interacción, los turnos de habla e indican al interlocutor su respuesta (continuación, repetición, finalización), al mismo tiempo que aportan señales informando los momentos de la conversación. Por ello, diversos autores señalan que la mirada es el regulador más importante para tomar el turno o acompañar el habla (Albadejo, 2007; Bull, 1994; Nobe, 2000; Salgado y Otero, 2020).

Las señales de afecto, son las expresiones de los estados de ánimo reflejados en la gestualidad facial y corporal, allí se pueden observar de forma clara estados de ansiedad o tensión emotiva, es por ello que, los movimientos del cuerpo muestran la intensidad de la emoción que se siente y la cara refleja el tipo de emoción. En consecuencia, son el cuerpo y el rostro los que transmiten las emociones y sentimientos, por lo tanto, se pueden reconocer señales afectivas intencionales o no intencionales (Fossa, 2019; Ricci y Cortessi, 1980; Rulicki y Cherny, 2012). Lo cierto es que, la cara es una zona especializada en comunicar emociones y actitudes, es decir, es la parte del cuerpo más compleja y significativa en la comunicación, puesto que es el área más observada en la interacción social, debido a que es el principal sistema de señales emocionales (Barrett et al., 2019).

En esta dirección, Ekman y Friesen (1969), señalan que el rostro es la sede primaria de la expresión de las emociones y la denominan como exhibidor del afecto, pues permite la interacción humana por medio de la expresión de los estados emocionales que, a su vez, proporcionan información y comprensión para actuar como meta-comunicación al modificar, complementar o comentar lo que se está diciendo. En general, algunos comportamientos como el sonreír, abrazar o dar una palmada, envían mensajes de empatía y crean dinámica y clima emocional entre los individuos (Argyle y Williams, 1969; Barret et al., 2019; Caballo, 1993; Ramallo, 2019; Ricci y Cortessi, 1980).

Por último, los adaptadores funcionan como gestos no intencionales, son vestigios de ciertos patrones conductuales con una función adaptativa, es decir, emociones primitivas. Estos comportamientos gestuales son la señal que ayuda al comunicante a satisfacer necesidades personales y a adaptarse a la interacción establecida (Albaladejo, 2007). Por su parte Ekman y Frisen (1969), señalan tres tipos de adaptadores: los gestos auto adaptadores, como los movimientos del cuerpo y el rostro, los cuales están relacionados con el cuidado físico y la adaptación a condiciones del ambiente; 
los adaptadores dirigidos a otros, es decir, estrategias de interacción prototípicas y elementales como cortejo y ataque y; por último, los adaptadores dirigidos a objetos, como las rutinas conductuales que se realizan con objetos del entorno. Por consiguiente, queda definido que estos gestos espontáneos acompañan el habla y son muy activos en el aprendizaje, al involucrar procesos de autorregulación referentes a operaciones cognitivo-emocionales (Singer y Goldin-Meadow, 2005).

Lo anterior, pone de manifiesto el valor de las habilidades comunicativas y la interpretación kinestésica y emocional, como una herramienta de suma importancia en las relaciones interpersonales y un aspecto clave en los procesos de enseñanza y de aprendizaje (Andrä et al., 2020; Barret et al., 2019; Damsgaard et al., 2020; Denervaud et al., 2020; Lockwood et al., 2016; Santiago et al., 2019). En consecuencia, el presente estudio buscó analizar la relación de las emociones con los procesos comunicativos no verbales de las niñas y niños, así como develar los comportamientos gestuales que se expresan en el aula infantil, los cuales configuran e inciden en el clima emocional educativo, dado que la etapa de iniciación escolar ha sido definida como un periodo en el cual crecen las interacciones sociales y las bases para la gestión de emociones, ya que aparecen las relaciones y vínculos entre compañeros (Usler et al., 2020).

\section{Método}

La presente investigación se fundamentó en la metodología observacional, estrategia que permite describir la realidad con objetividad a partir de la recolección sistemática de datos para analizarlos y obtener conocimientos válidos de acuerdo a los objetivos planteados (Anguera, 1986; Anguera et al., 2011; Anguera et al., 2013), lo que la hace una metodología pertinente para el estudio del comportamiento humano (Anguera, 2010), en gran medida, porque integra un criterio procedimental y uno sustantivo, el primero orientado a la recogida de datos directa, visual, digital y auditiva de los hechos y, el segundo, el sustantivo, permite la generación de respuesta a diversos objetivos (Anguera y Hernández-Mendo, 2013), generando una complementariedad entre lo cualitativo y lo cuantitativo (Anguera, 2003, 2004). Para ello, se realiza un registro organizado que permite una codificación de cada conducta (Castañer et al., 2013; Del Giacco et al., 2020).

Por consiguiente, la metodología observacional asume una perspectiva mixta ya que en la primera fase adopta una vertiente cualitativa y en la segunda, una de tipo cuantitativo (ver figura 1), es decir, que se realiza un proceso en el que el registro empieza siendo narrativo, para pasar a ser descriptivo, luego semi-sistematizado y por último sistematizado, por lo tanto, se fortalecen los análisis y resultados obtenidos. En el mismo sentido, tal y como señalan Johnson y Onwuegbuzie (2004), esta metodología se puede con- siderar como un estudio mixto con igualdad de estatus y de orden secuencial.

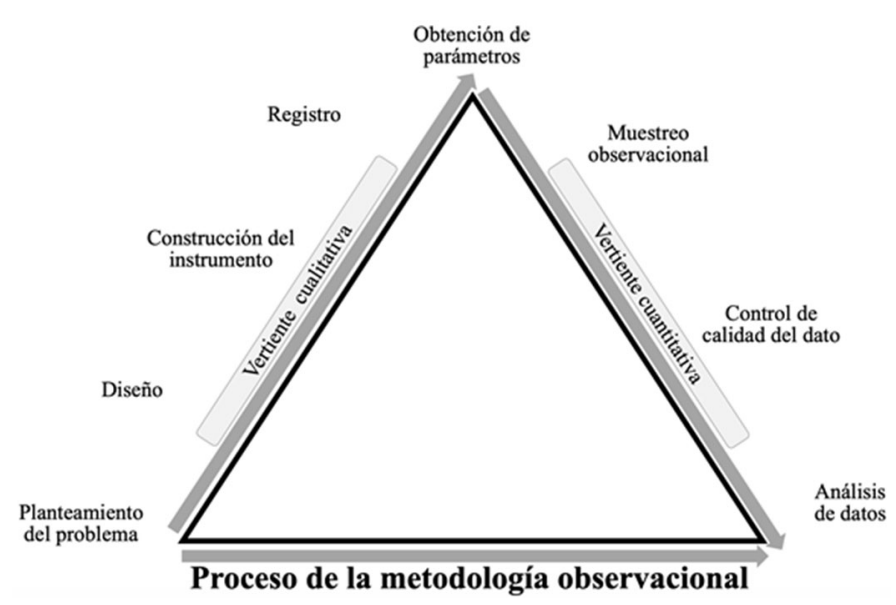

Figura 1.

Complementariedad cualitativa-cuantitativa en la metodología observacional (Anguera, 2004)

De igual manera, de acuerdo a los planteamientos de Anguera-Argilaga et al. (2007), en la metodología observacional se pueden implementar ocho diseños (ver figura 2), siendo el que se utilizó en la presente investigación el de tipo: Seguimiento/Nomotético/Multidimensional (S/N/M), es decir, de seguimiento debido a la temporalidad, puesto que implicó varias sesiones; nomotético, por la pluralidad de unidades que se estudiaron de manera independiente y; multidimensional, por los diversos niveles de respuesta (Anguera et al., 2001).

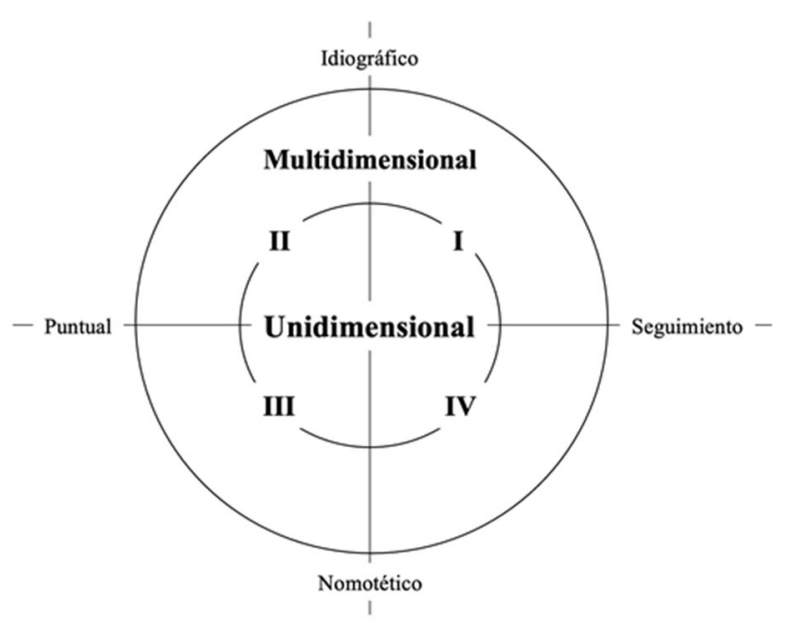

Figura 2.

Diseños Observacionales (Anguera, 2001; Anguera et al., 2001) 
Para la preparación de la observación se tuvieron en cuenta tres aspectos fundamentales: a. Observación Exploratoria, la cual fue de tipo asistemático y buscó estructurar de manera adecuada el problema de investigación; disminuir el sesgo de reactividad; fortalecer el nivel de entrenamiento del observador y; el análisis de informaciones anecdóticas para la toma de decisiones; b. Requisitos Idóneos, referidos a aspectos que permitieron no incurrir en carencias o errores metodológicos, para ello, se tuvo en cuenta el mantenimiento de la constancia intersesional, es decir, lograr la mayor homogeneidad en las diferentes sesiones, para ello se establecieron los criterios que se tenían que cumplir en cada sesión para que fuera válida. De igual manera, el mantenimiento de la constancia intrasesional, la cual implicó la determinación respecto a los datos que se podian tener en cuenta o no durante el registro de la clase. También, la elaboración de un plan o agenda relativo a la sucesión de actividades a desarrollar a lo largo del proceso, fase exploratoria, diseño del estudio, elaboración del instrumento de observación, registro y control de calidad del dato, análisis de información, interpreración de resultados y elaboración del informe, por último, la identificación de la sesión de observación, que requirió el análisis del contexto en cuanto al entorno físico, actividades realizadas, nivel social de los niños e información institucional y; c. Reducción del Sesgo, centrado en la reactividad, para lo cual se realizaron diversas actividades para que los niños integraran la presencia de la cámara de video como un factor natural y cotidiano. Además de ello, la expectancia, se abordó con ejercicios preliminares realizados en la fase exploratoria, para reducir las previsiones y anticipaciones de conductas.

\section{Participantes}

Hicieron parte del estudio estudiantes del grado de transición I, pertenecientes a la Institución Educativa oficial Instituto Técnico Gonzalo Suárez Rendón de la ciudad de Tunja (Colombia), haciendo uso del muestreo intencional (Flick, 2015). El total de participantes de transición fue ( $n$ $=21)$, siendo niñas $(n=8)$ y niños $(n=13)$, la edad media fue de 5,48 años $(D T=0,512)$, distribuidos en 5 años $(n=$ 11) y 6 años $(n=10)$.

\section{Instrumento}

Tal y como señala Anguera-Argilaga et al. (2007) en la metodología observacional no se busca elicitar la respuesta sino la construcción de un instrumento ad hoc a partir del volcado de los datos. Para la presente investigación se hizo uso del sistema de categorías y el formato de campo, siendo este último, una antigua técnica de registro (Weick, 1968), que retoma Anguera-Argilaga (1979) y que en la actualidad se considera como un instrumento de observación (Anguera, 2001). En cualquier caso, los instrumentos no estándar posibilitan la transformación de los datos, su control de calidad y la aplicación de las técnicas analíticas pertinentes. El instrumento ad hoc permite "la transcripción de la representación de la realidad por parte del observador mediante la utilización de códigos determinados y que se materializan en un soporte físico que garantiza su prevalencia" (Anguera et al., 1993, p. 81).

Tabla 1.

Categoría y subcategorías establecidas

\begin{tabular}{lll}
\hline Categoría & Subcategorías & Descripción \\
\hline
\end{tabular}

Emblemas Está referido a los comportamientos que no son vocales y tienen traducción implícita, es decir están cargados de significado (Cestero, 1998).

Ilustradores

Comportamientos gestuales

Reguladores

Adaptadores

Señal de afecto
Los ilustradores son gestos que acompañan e ilustran el mensaje que se está comunicando mediante el uso de la corporalidad, la gestualidad y la entonación, permiten señalar físicamente relaciones espaciales, imágenes o movimientos (Shablico, 2012).

Los reguladores, son movimientos y comportamientos que median las interacciones sociales y permiten mantener la comunicación gracias a sus pausas, silencios, cambios de entonación lo cual genera interés en los interlocutores (Domínguez, 2009).

Son gestos no intencionales, que cumplen una función adaptativa, dado que suelen expresarse por medio de las emociones primitivas, con el fin de adaptarse a la interacción y situación establecida, pueden estar dirigidos hacia el mismo cuerpo, objetos u otra persona (Albaladejo, 2007)

Son las expresiones de los estados de ánimo reflejados en la gestualidad facial y los gestos corporales dirigidos a otra persona y los cuales se pueden reconocer según sus señales afectivas (Rulicki y Cherny, 2012). 
Es relevante señalar que, se requiere para estos instrumentos el tener soporte en el marco teórico, así como gran flexibilidad para integrar las nuevas categorías emergentes de la realidad. En cualquier caso, los instrumentos no estandarizados se establecen como técnicas de registro que en la actualidad se utilizan con gran frecuencia en la metodología observacional y se caracterizan por poseer un sistema abierto, multidimensional, de código múltiple y autor regulable (Anguera, et al, 2018; Blanco et al., 2001). Dentro de la categoría y subcategorías establecidas para este estudio se encuentran las siguientes (ver tabla 1).

\section{Procedimiento}

Fase Pasiva, Exploratoria o Precientifica. En esta fase es absolutamente imprescindible preparar la observación activa o científica, permitiendo delimitar el problema de forma precisa reduciendo los sesgos y la reactividad, así como mejorar el entrenamiento del observador que posibilita obtener un bagaje completo de información para la toma de decisiones.

De igual manera, se abordó el control de los sesgos que pudiesen presentarse, como por ejemplo la reactividad, es decir, la alteración de la naturaleza (simple y recíproca); la expectancia, la cual surge en el observador en forma de previsiones y/o anticipaciones de conductas no contextualizadas de carácter técnico, dentro de los cuales se cuidó el ángulo incorrecto, la planificación incorrecta del muestreo observacional y los fallos técnicos, además de ello, se buscó establecer la ecuación funcional: Observación=Percepción+Interpretación+Conocimiento previosSesgos. De igual forma, en esta fase se obtuvo el aval institucional, así como el consentimiento informado por parte de los padres de familia para la grabación de las sesiones.

Fase Activa o Científica. En esta fase se realizó la observación sistemática, la cual incluyó el registro descriptivo, el registro semi-sistematizado y finalmente el registro sistematizado, establecido a través de códigos y listas de rasgos estructurados en una matriz de códigos de categorías y subcategorías.

Recolección de datos. Se realizó el registro fílmico de ocho clases, de las cuales se excluyeron dos al no cumplir con los criterios establecidos respecto al mantenimiento de la constancia intersesional, es decir, sesiones en las cuales hubo interrupción por actividades fuera del salón que implicaron la no grabación de la totalidad de la clase. Hay que resaltar, que cada sesión grabada tuvo una duración de dos horas, fraccionadas en dos momentos, una hora de juego libre y una hora de clase estructurada.

Transcripción de la información. Al volcar la información en el instrumento ad-hoc, se determinó la presunción de exhaustividad al completar el análisis de la tercera sesión, debido a que no aparecieron nuevas conductas, por este motivo, el estudio se llevó a cabo bajo el análisis de estas tres primeras sesiones.

\section{Análisis de los datos}

El análisis de los datos fue desarrollado mediante técnicas de análisis cuantitativo, utilizando el programa informático SPSS para Windows (versión 24.0) un software para el análisis estadístico de la investigación social y educativa. En este sentido los análisis implementados fueron:

- Estadísticos descriptivos: frecuencia, porcentaje, media y desviación típica de las diferentes variables objeto de estudio.

- $\quad$ Análisis de frecuencias, mediante el estadístico Chi cuadrado $\left(\chi^{2}\right)$.

También es importante señalar que los resultados obtenidos se editaron en el programa Microsoft Office Word 2010 para optimizar su presentación y comprensión.

\section{Resultados}

Se analizaron un total de 686 gestos relacionados con acciones de comunicación emocional no verbal. En primer lugar, se estableció si en cada situación comunicativa intervenía un solo gesto o una secuencia de gestos (ver tabla 2).

Tabla 2.

Número de gestos en las acciones comunicativas

\begin{tabular}{lcc}
\hline Gesto/Secuencia & Frecuencia & Porcentaje \\
\hline Un gesto & 210 & 30,6 \\
Secuencia & 476 & 69,4 \\
\hline
\end{tabular}

En el mismo sentido, la figura 3 establece el número de gestos que intervinieron en cada una de las acciones comunicativas analizadas, los cuales estuvieron en el rango de 1 a 4 gestos en cada secuencia.

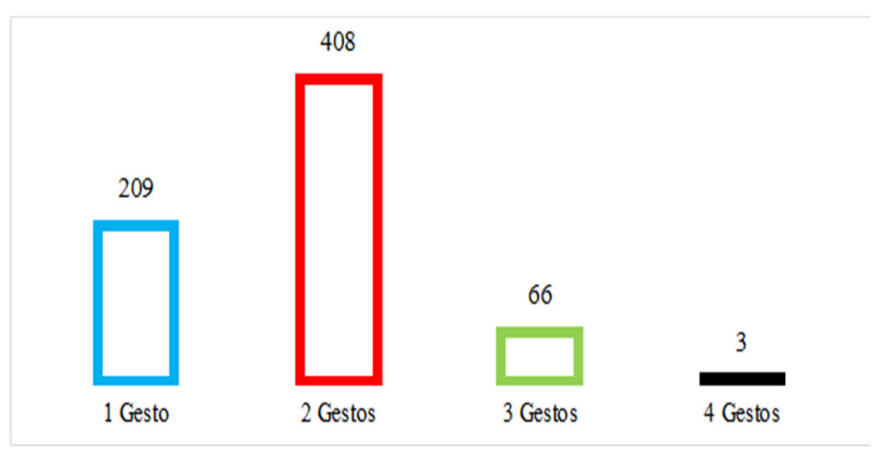

Figura 3.

Frecuencia del número de gestos en las acciones comunicativas 
En el mismo sentido, la figura 4 presenta la frecuencia que emergió en cada uno de los cinco tipos de gestos que se analizaron (Adaptador, Emblema, Ilustrador, Regulador, Señal de Afecto).

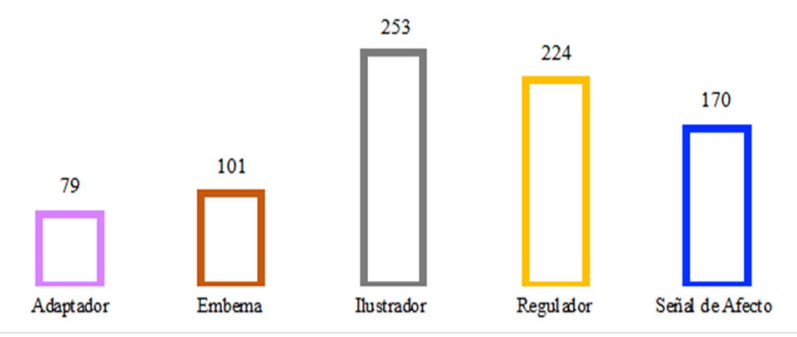

Figura 4.

Frecuencia de cada uno de los gestos en las secuencias comunicativas

Además de ello, se estableció el porcentaje en que cada gesto funcionó como inicial en cada acción comunicativa (figura 5).

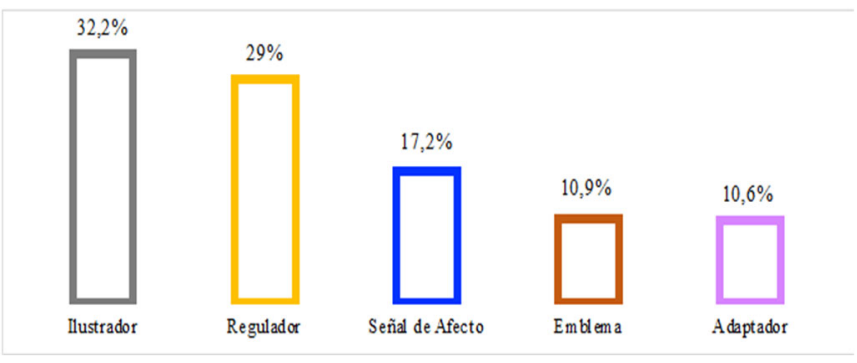

Figura 5.

Porcentaje del gesto inicial de cada secuencia

El análisis de frecuencias en cuanto al gesto inicial en las situaciones registradas en cada sesión en función del sexo del emisor, se presenta en la tabla 3 , no hallándose diferen-

Tabla 3 .

Frecuencias y prueba Chi-cuadrado del gesto inicial en función del sexo del emisor

\begin{tabular}{|c|c|c|c|c|c|c|}
\hline Sexo Emisor & Adaptador & Emblema & Ilustrador & Regulador & $\begin{array}{l}\text { Señal de } \\
\text { Afecto }\end{array}$ & $\mathrm{Chi}^{2}$ \\
\hline Niña & 34 & 37 & 83 & 73 & 48 & 5,516 \\
\hline$\%$ Sexo Emisor & $12,4 \%$ & $13,5 \%$ & $30,2 \%$ & $26,5 \%$ & $17,5 \%$ & \\
\hline$\%$ Gesto Inicial & $46,6 \%$ & $49,3 \%$ & $37,6 \%$ & $36,7 \%$ & $40,7 \%$ & \\
\hline Niño & 39 & 38 & 138 & 126 & 70 & \\
\hline \% Sexo Emisor & $9,5 \%$ & $9,2 \%$ & $33,6 \%$ & $30,7 \%$ & $17,0 \%$ & \\
\hline$\%$ Gesto Inicial & $53,4 \%$ & $50,7 \%$ & $62,4 \%$ & $63,3 \%$ & $59,3 \%$ & \\
\hline
\end{tabular}

De igual forma, se analizaron las frecuencias del gesto inicial que se generó en cada una de las sesiones en función de la edad del emisor (tabla 4). En esta variable tampoco se hallaron diferencias estadísticamente significativas.

Tabla 4.

Frecuencias y prueba Chi-cuadrado de las sesiones en función de la edad del emisor

\begin{tabular}{|c|c|c|c|c|c|c|}
\hline Edad Emisor & Adaptador & Emblema & Ilustrador & Regulador & $\begin{array}{l}\text { Señal de } \\
\text { Afecto }\end{array}$ & $\mathrm{Chi}^{2}$ \\
\hline 5 años & 50 & 48 & 115 & 107 & 70 & 8,749 \\
\hline$\%$ Edad Emisor & $12,8 \%$ & $12,3 \%$ & $29,5 \%$ & $27,4 \%$ & $17,9 \%$ & \\
\hline$\%$ Gesto Inicial & $68,5 \%$ & $64,0 \%$ & $52,0 \%$ & $53,8 \%$ & $59,3 \%$ & \\
\hline 6 años & 23 & 27 & 106 & 92 & 48 & \\
\hline$\%$ Edad Emisor & $7,8 \%$ & $9,1 \%$ & $35,8 \%$ & $31,1 \%$ & $16,2 \%$ & \\
\hline$\%$ Gesto Inicial & $31,5 \%$ & $36,0 \%$ & $48,0 \%$ & $46,2 \%$ & $40,7 \%$ & \\
\hline
\end{tabular}




\section{Discusión}

El presente estudio analizó desde una metodología observacional el movimiento del cuerpo, dado que este, con sus procesos dinámicos expresa emociones, percepciones, motivaciones y lazos interpersonales, los cuales permitieron evidenciar una frecuencia estable de situaciones comunicacionales en cada una de las sesiones registradas. Es por ello, que el ambiente escolar permite configurar interacciones para la vida y el desarrollo integral en todos los niveles de educación, aún más en los niños, creando proximidad, sentido de grupo y el establecimiento de una comunicación emocional asertiva, en la que el cuerpo tiene protagonismo, capacidad significativa y discurso emocional que favorece los procesos comunicativos en el aula de clase. Así mismo, posibilita reconocer al otro y su forma de relacionarse, es decir, es reflejo de un clima emocional esencial para favorecer los aprendizajes y las relaciones escolares, lo cual implica un mejor desempeño social y académico (Albaladejo, 2007; Buitrago-Bonilla y Cárdenas-Soler, 2017; Gallo, 2009; Gordillo et al., 2015; Hargreaves, 2000; Herrera et al., 2017; Lapierre y Aucoutourier, 1977; Moreno y Gálvez, 2010; Igualada et al., 2015; SalzbergerWittenberg et al., 1983).

También es preciso señalar que, en concordancia con otros estudios (Carlson y Wang, 2007; García y Tully, 2020; Gordillo et al., 2015; Ip et al., 2021; Koch y Pollatos, 2015; Moffitt et al., 2011; Salisch et al., 2013), la presente investigación evidenció la habilidad de las niñas y niños para identificar, categorizar y comprender distintas expresiones emocionales, así como para responder y actuar de acuerdo con ellas. Además, se encontró que los participantes mostraron gran variabilidad en cuanto al número de veces en que iniciaban una acción comunicativa, así como en el número de expresiones que integraban en la secuencia comunicativa, éstas variaciones dan cuenta, en alguna medida, de la configuración intrapersonal de cada uno, las cuales, como se ha estudiado, están determinadas por sus experiencias familiares, sociales y escolares, por lo que configuran la singularidad de cada uno de ellos. Esta perspectiva, se relaciona con los planteamientos de Davidson y Begley (2012), quienes enfatizan en que el cerebro de cada persona cuenta con un perfil emocional que se puede observar, reconocer y analizar, el cual, además, determina las diferencias intrapersonales que caracterizan a cada sujeto, motivo por el cual, es preciso señalar que, la experiencia emocional es subjetiva y está mediada por sistemas neuronales (Perlovsky y Schoeller, 2019; Kleinginna y Kleinginna, 1981).

En cuanto a los comportamientos gestuales (ilustradores, reguladores, señales de afecto, emblemas y adaptadores), se hizo evidente que cada uno de los gestos expresados y sus significados, fueron establecidos por las secuencias en que se dieron, lo cual concuerda con el planteamiento de Mondloch (2012), quien explicita que el significado de cada gesto es definido por la combinación de muchos significados, aún más, cuando existen dos o más gestos sucesivos, así, la integración de varios gestos en la comunicación de las personas hace visible sus recuerdos y sus pensamientos internos (Barret et al., 2019; Fossa, 2019).

De igual manera, se encontró que cada acción comunicativa se estructuró de acuerdo al número gestos que la componía y que dichas secuencias pusieron de manifiesto que la comunicación emocional de las niñas y niños participantes en el estudio es sencilla y que aunque no se evidenciaron diferencias estadísticamente significativas, sí se pudieron encontrar algunos pequeños cambios entre los participantes de cinco y seis años, ya que algunos de los primeros se comunicaban con una secuencia de dos gestos, mientras que algunos de los infantes de seis años lo hacían con secuencias de tres a cuatro gestos. Estos hallazgos están en la misma línea de Carlson y Wang (2007), al igual que de Kachel et al. (2021), quienes señalan que se presenta una relación directamente proporcional entre el aumento de edad y la manifestación de expresiones emocionales más complejas e intensas. De igual forma, algunos estudios conductuales y neurológicos afirman que la capacidad para discriminar, diferenciar y conceptualizar las emociones a través de las expresiones faciales, está relacionada con la edad, dado que surge desde los primeros años de vida, específicamente durante el primer año de edad y su desarrollo se extiende de manera progresiva (Esteve-Gibert et al., 2019).

Otros estudios del comportamiento humano, han puesto de manifiesto que los infantes de cinco años pueden integrar palabras y gestos a la par con las tareas realizadas, lo cual en la presente investigación justifica la alta presencia de gestos ilustradores y reguladores en los procesos comunicativos, los cuales tal y como se había señalado, se constituyen como una forma de aprendizaje socioemocional (AlZoubi et al., 2012). A esto se suma que, al analizar los gestos iniciales en las secuencias, fueron los ilustradores los que tuvieron mayor presencia, permitiendo ilustrar, acentuar y complementar el contenido del mensaje o la entonación del mismo (Shablico, 2012).

En esta dirección, Manwaring et al. (2017) realizaron una revisión de 19 estudios sobre los gestos ilustradores, específicamente deícticos en niños autistas, encontrando que estos gestos eran usados en la comunicación al indicar objetos, personas y lugares próximos a su entorno inmediato, aspecto concordante con la presente investigación, ya que los gestos usados por los niños en la comunicación con sus compañeros y la docente, permitió relacionar la ubicación de juguetes, material didáctico y útiles escolares. Lo cierto es que estos gestos son considerados mensajes explícitos 
que pueden ser inconscientes pero que se relacionan con lo hablado y las tareas realizadas (Dupertuis y Moro, 2016). Así mismo, los ilustradores requieren amplia comprensión por parte de los interlocutores para lograr una comunicación asertiva (Rodríguez et al., 2015). Por esta razón, los niños de entre tres y cinco años, por lo general, están aprendiendo gradualmente a comprender la información transmitida a través de gestos relacionados con el habla (Minto et al., 2018; Stanfield et al., 2014).

Los gestos que se ubicaron en segundo lugar fueron los reguladores, los cuales son encargados de sincronizar el diálogo, los turnos del habla y la interacción, al respecto Pouw et al. (2020) señalan que la sincronía gesto-habla se realiza de acuerdo a los mensajes que viajan a través del cuerpo, en mayor proporción cabeza, rostro, manos y por supuesto la mirada como reguladora de la comunicación, por ello, se convierte en el gesto más importante para tomar el turno del habla. Además de ello, fue evidente de manera preponderante la expresión de gestos autorreguladores, que son los relacionados con el propio cuerpo, cabello, cabeza, cuello y uñas, seguidos de los gestos reguladores dirigidos a objetos, específicamente a los útiles escolares. Al respecto, se puede plantear una diferencia con lo encontrado por Maricchiolo et al. (2009), quienes proponen que los gestos que están vinculados al habla afectan la evaluación del mensaje y su juicio de manera más positiva que los gestos no vinculados al habla. No obstante, en el presente estudio la comprensión de los comportamientos gestuales en los niños llevó a determinar que la apreciación que hacían de los gestos no vinculados al habla, en muchas ocasiones, era más positiva y complementaba su discurso. En este sentido, es relevante comentar los planteamientos de Demir-Lira et al (2018), quienes enfatizan en la integración gesto-habla y en la activación cerebral que se presenta en los infantes de 8 a 11 años, la cual posibilita que el gesto proporcione información complementaria que no está presente en el discurso.

Para terminar, es importante señalar que las señales de afecto tuvieron una presencia importante en la comunicación de los participantes, y que los gestos que presentaron menor presencia fueron los emblemas y los adaptadores. En cualquier caso, la presente investigación permitió evidenciar un amplio número de gestos en los procesos comunicativos de los participantes, lo cual no solo está asociado al desarrollo natural de las niñas y niños, sino que puede estar incidido por la apuesta que hace la Institución Educativa respecto al desarrollo humano y emocional de sus alumnos para fortalecer los procesos de enseñanza y de aprendizaje, integrando la configuración de un adecuado clima de aula y la generación de relaciones interpersonales positivas, aspectos que se reafirman por diversos estudios (Buitrago y Herrera, 2013; Gutiérrez-Torres y BuitragoVelandia, 2019; Herrera et al., 2016; Palomera et al.,
2019). Lo cierto es que, los comportamientos gestuales están relacionados con procesos de conceptualización y aprendizaje de sus significados comunicativos (EsteveGibert et al., 2019; Lüke et al., 2020; Mumford y Kita, 2014).

\section{Conclusiones}

Es necesario que en la escuela se adquieran y se enseñen habilidades para el reconocimiento de las emociones, como proceso transversal y progresivo que aporta a los procesos comunicativos y a la gestión emocional, integrando la corporalidad para reconocer, expresar, dirigir y gestionar las emociones propias y de los demás, tal y como lo proponen Mayer y Salovey (1993; 1997; 2009), integrando, como se ha señalado, la evaluación verbal y no verbal para fortalecer el desarrollo intrapersonal, interpersonal y social (Barret et al., 2019; Buitrago y Herrera, 2013; Davidson y Begley, 2012; Denervaut et al., 2020; Gil-Olarte, 2006; Pena et al., 2016), así como el bienestar (Herrera et al., 2015; Palomera, 2017).

De otro lado, aunque la educación del cuerpo ha sido abordada desde disciplinas como la antropología, la sociología, la psicología y la educación, es necesario resaltar que aún requiere mayor énfasis en su potencial comunicativo, sobre todo en los contextos escolares, en donde, este potencial se ha excluido, de alguna manera, del proceso de aprendizaje, por lo que se hace necesario abordar el cuerpo desde la experiencia subjetiva y la comunicación. Por consiguiente, la escuela debe considerarse y concebirse como un espacio en el cual se fortalezcan las habilidades comunicativoemocionales de los estudiantes, las cuales se deberían estar integradas en los currículos.

Así mismo, el desarrollo de habilidades socioemocionales debe considerarse como motor de la educación que debe tener en cuenta al cuerpo, debido a que inciden en lo físico, biológico, moral, social y cultural, tanto en espacios escolares como extraescolares (Cespedes, 2020). De esta manera, la educación llevará a cabo el proceso de transformación personal y comunitaria, impactando positivamente en el desarrollo de capacidades intelectuales, sociales y emocionales (Buitrago et al., 2019; Palomera et al., 2019), por ello, se hace de vital importancia que desde la infancia se propicie de manera efectiva la interacción con los demás y con el entorno para fortalecer el desarrollo de habilidades comunicativas, de escucha, de empatía, de asertividad, así como de intuición y responsabilidad social, aspectos fundamentales para el desarrollo y bienestar de las niñas y niños (Calkins, 2010).

También, es imprescindible enfatizar en la importancia del cuerpo en la educación, las relaciones escolares y las prácticas pedagógicas, porque permite integrar el sentir y el pensar con la cultura y la educación, además de establecer 
la educación como una experiencia de comunicación emocional (Gallo, 2017), desde una perspectiva amplia que también vincule al maestro, su gestualidad, corporalidad y afectividad (Extremera et al., 2019; Palomera et al., 2017, 2019).

Finalmente, se destaca la importancia de capacitar y desarrollar procesos que permitan a los maestros la identificación y análisis de los comportamientos gestuales de sus estudiantes, como una herramienta que favorece la gestión del clima de aula, la resolución de conflictos y la relación con los estudiantes (Denervaud et al., 2020; Extremera et al., 2019; Gutiérrez-Torres y Butirago-Velandia, 2019; Pena et al., 2019). De igual modo, se hace indispensable continuar indagando sobre las manifestaciones gestuales y emocionales en la infancia dentro y fuera del aula, para aportar a los procesos educativos desde perspectivas culturales y emocionales diversas.

\section{Limitaciones del Estudio}

Quizá la mayor limitación está relacionada con haber utilizado una muestra de una sola institución educativa, ya que, si bien es cierto que al hacerlo se pudo conocer la realidad en cuanto a la comunicación emocional no verbal de sus participantes, limitó la posibilidad de conocer y contrastar la realidad de otros contextos educativos. De otro lado, al realizar el registro con una única cámara de video, se generaron, como es lógico, algunos sectores de inobservabilidad, los cuales se hubiesen podido reducir con la integración de otras cámaras. En cualquier caso, la presente investigación establece un punto de partida importante para seguir estudiando la expresión emocional y la comunicación infantil en la escuela y deja una serie de interrogantes y perspectivas de indagación para seguir profundizando en los hallazgos obtenidos y que se pueden complementar con el abordaje de otras perspectivas metodológicas, otras técnicas e instrumentos de recolección de información y nuevos análisis.

El presente estudio se desarrolló en la línea de investigación Emociones y Educación del Grupo de Investigación Cacaenta de la Universidad Pedagógica y Tecnológica de Colombia

\section{Referencias}

Albaladejo, M. (2007). La Comunicación más allá de las palabras: qué comunicamos cuando creemos que no comunicamos. Barcelona: Graó.

Alibali, M.W. y Kita, S. (2010). Gesture highlights perceptually present information for speakers. Gesture, 10 (1), 3-28. https://di.org/10.1075/gest.10.1.02ali

AlZoubi, O., D'Mello, S. K. y Calvo, R.A. (2012). Detecting naturalistic expressions of nonbasic affect using physiological signals. IEEE Transactions on Affecti- ve Computing, 3(3), 298-310.

Andrä, C., Mathias, B., Schwager, A., Macedonia, M. y von Kriegstein, K. (2020). Learning foreign language vocabulary with gestures and pictures enhances vocabulary memory for several months postlearning in eight-year-old school children. Educational Psychology Review, 32, 815-850. https:// doi.org/10.1007/s10648-020-09527-z

Anguera-Argilaga, M.T. (1979). Observational typology. Quality and Quantity, 13, 449-484. https:// doi.org/10.1007/BF00222999

Anguera-Argilaga, M.T., Magnusson, M. y Jonsson, G. (2007). Instrumentos no estándar: planteamiento, desarrollo y posibilidades. Avances en Medición, 5, 63-82.

Anguera, J., Puente, C., Borja, C., Montero, R. y Soler, J. (2001). Small and high-directivity bow-tie patch antenna based on the Sierpinski fractal. Microwave and Optical Technology Letters, 31(3), 239-241.

Anguera, M.T. (1986). La investigación cualitativa. Educar, 10, 23-50.

Anguera, M.T. (2001). Cómo apresar las competencias del bebé mediante una aplicación de la metodología observacional. Contextos Educativos. Revista de Educación, (4), 13-34.

Anguera, M.T. (2003). La observación. En C. Moreno (Ed.), Evaluación psicológica. Concepto, proceso y aplicación en las áreas del desarrollo y de la inteligencia (pp. 271-308). España: Sanz y Torres.

Anguera, M.T. (2004). Posición de la metodología observacional en el debate entre las opciones metodológicas cualitativa y cuantitativa. ¿Enfrentamiento, complementariedad, integración? Psicologia em Revista, 10 (15), 13-27.

Anguera, M.T. (2010). Posibilidades y relevancia de la observación sistemática por el profesional de la Psicología. Papeles del Psicólogo, 31(1), 122-130.

Anguera, M. ., Behar, J., Blanco, A., Carreras, M.V., Losada, J.L., Quera, V. y Riba, C. (1993). Glosario. En M. T. Anguera (Ed.). Metodología observacional en la investigación psicológica, vol. II (pp. 587-617). Barcelona: Promociones y Publicaciones Universitarias.

Anguera, M.T., Blanco-Villaseñor, A. y Losada, J.L. (2001). Diseños observacionales, cuestión clave en el proceso de la Metodología Observacional. Metodología de las Ciencias del Comportamiento, 3(2), 135-160. 
Anguera, M.T., Blanco-Villaseñor, A., Losada, J.L. y Portell, M. (2018). Pautas para elaborar trabajos que utilizan la metodología observacional. Anuario de Psicología, 48(1), 9-17. https://doi.org/10.1016/ j.anpsic. 2018.02 .001

Anguera, M.T. y Hernández-Mendo, A. (2014). Metodología observacional y psicología del deporte: Estado de la cuestión. Revista de Psicología del Deporte, 23(1) 103-109.

Anguera, M.T. y Mendo, A.H. (2013). La metodología observacional en el ámbito del deporte Revista de Ciencias del Deporte, 9(3), 135-160.

Anguera, M.T., Villaseñor, A.B., Mendo, A.H. y López, J.L.L. (2011). Diseños observacionales: ajuste y aplicación en psicología del deporte. Cuadernos de Psicología del Deporte, 11(2), 63-76.

Argyle, M. (1975). Bodily Communication. Londres: Methuen and Co.

Argyle, M. y Williams, M. (1969). Observer or observed? A reversible perspective in person perception. Sociometry, 396-412. https://doi.org/ 10.2307/2786543

Barrett, L.F., Adolphs, R., Marsella, S., Martínez, A.M. y Pollak, S.D. (2019). Emotional Expressions Reconsidered: Challenges to Inferring Emotion from $\mathrm{Hu}$ man Facial Movements. Psychological Science in the Public Interest, 20(1), 1-68. https:// doi.org/10.1177\%2F1529100619832930

Belío-Apaolaza, H.S. (2018). Aprendizaje y evaluación de la comunicación no verbal en ELE. Propuesta teórica y estudio empírico sobre los gestos emblemáticos [Tesis doctoral, Universidad de Salamanca]. https:// gredos.usal.es/handle/10366/140308

Belío-Apaolaza, H.S. (2019). La naturaleza lingüísticocomunicativa de los gestos emblemáticos. Una propuesta de representación. Círculo de Lingüística Aplicada a la Comunicación, 80, 115-134. https:// doi.org/10.5209/clac.66603

Białek, A., Białecka-Pikul, M., Filip, A. y Broda, M. (2018) La relevancia es importante. El uso de gestos deícticos relevantes e informativos por parte de niños de año y medio como factor predictor de las capacidades lingüísticas a los dos años. Infancia $y$ Aprendizaje, 41(4), 674-701. https:// doi.org/10.1080/02103702.2018.1514710

Blanco, A.B., Losada, J.L. y Anguera, M.T. (2001). Diseños observacionales, cuestión clave en el proceso de la metodología observacional. Metodología de las Ciencias del Comportamiento, 3(2), 135-160.
Buitrago, R.E. y Herrera, L. (2013). Matricular las Emociones en la Escuela, una Necesidad Educativa y Social. Praxis y Saber, 8(4), 87-108.

Buitrago-Bonilla, R.E. y Cárdenas-Soler, R.N. (2017). Emociones e identidad profesional docente: relaciones e incidencia. Praxis y Saber, 8(17), 225-247. https:// doi.org/10.19053/22160159.v8.n17.2018.7208

Buitrago, R.E., Herrera, L. y Cárdenas, R.N. (2019). Coeficiente emocional en niños y adolescentes de Boyacá, Colombia. Estudio comparativo. Praxis y Saber, 10(24), 45-68. https:// doi.org/10.19053/22160159.v10.n25.2019.10002

Bull, P. (1994). On identifying questions, replies, and nonreplies in political interviews. Journal of Language and Social Psychology, 13(2), 115-131.

Bunglowala, A. y Bunglowala, A. (2015). Nonverbal communication: An integral part of teaching learning process. International Journal of Research in Advent Technology, 371-375.

Burgoon, J.K., Guerrero, L.K. y Manusov, V. (2016). Nonverbal communication. Londres: Routledge.

Buriticá, D. y Saldarriaga, Ó. (2020). Voces infantiles y gestos pedagógicos en la escuela rural. Pedagogía y Saberes, 52, 23-35. https://doi.org/10.17227/ pys.num52-10025

Caballo, V.E. (1993). Relaciones entre diversas viedidas conductuales y de autoinforme de las habilidades sociales. Psicología Conductual, 1(1), 53-99.

Calkins, S.D. (2010). Commentary: Conceptual and methodological challenges to the study of emotion regulation and psychopathology. Journal of Psychopathology and Behavioral Assessment, 32(1), 92-95. https://doi.org/10.1007/s10862-009-9169-6

Carlson, S.M., y Wang, T.S. (2007). Inhibitory control and emotion regulation in preschool children. Cognitive Development, 22(4), 489-510. https://doi.org/ 10.1016/j.cogdev.2007.08.002

Carpena, A. y Darder, P., (2010). Educación emocional y salud y bienestar del profesorado. Barcelona: Graó.

Castañer, M., Camerino, O. y Anguera, M.T. (2013) Métodos Mixtos en la investigación de las Ciencias de la Actividad Física y el Deporte. Apuntes Educación Física y Deportes, (112), 11-16. http:// dx.doi.org/10.5672/apunts.2014-0983.es. $(2013 / 2) .112 .01$

Cespedes, G. (2020). Análisis de la inteligencia emocional en menores de familias problemáticas. Know and 
Share Psychology, 1(2), 51-62. http:// dx.doi.org/10.25115/kasp.v1i2.3171

Cestero, A. (1998). Estudios de comunicación no verbal. Madrid: Edinumen.

Cestero, A.M. (2014). Comunicación no verbal y comunicación eficaz. ELUA, 28, 125-150.

Cestero, A.M. (2015). La expresión del tabú: estudio sociolingüístico. Boletín de Filología, 50(1), 71-105. http://dx.doi.org/10.4067/S071893032015000100003

Cestero, A.M. (2016). La Comunicación no verbal: propuestas metodológicas para su estudio [Tesis de maestría, Universidad de Alcalá]. http:// hdl.handle.net/10017/25226

Cestero, A.M. (2017). La comunicación no verbal en el discurso persuasivo empresarial. Paralingüistica, (25), 124-145.

Cestero, A.M. (2018). Recursos no verbales en comunicación persuasiva: gestos. Zer, 23(44), 69-92. https:// doi.org/10.1387/zer.18130

Curioni, A., Knoblich, G. ., Sebanz, N. y Sacheli, L.M. (2020). The engaging nature of interactive gestures. Plos One, 15(4). https://doi.org/10.1371/ journal.pone. 0232128

Cyrulnik, B. (2020). De cuerpo y alma: neuronas y afectos: la conquista del bienestar. Barcelona: Editorial Gedisa.

Damsgaard, L., Elleby, S.R., Gejl, A.K., Bøgh, A.S., Bugge, A., Lundbye-Jensen, J., Poulsen, M., Nielsen, G. y Wienecke, J. (2020). Motor-Enriched Encoding Can Improve Children's Early Letter Recognition. Frontiers in Psychology, 11. https:/doi.org/ 10.3389/ fpsyg.2020.01207

Davidson, R.J. (2003a). La neurociencia de la emoción. En D. Goleman (Ed.), Emociones Destructivas, cómo entenderlas y superarlas (pp. 104-118). Barcelona: Kairós.

Davidson, R.J. (2003b). Seven sins in the study of emotion: Correctives from affective neuroscience. Brain and Cognition, 52, 129-132. https://doi.org/10.1016/ S0278-2626(03)00015-0

Davidson, R. y Begley, S. (2012). El perfil emocional de tu cerebro. Barcelona: Ediciones Destino.

De Santiago, J. (2005): Principios de comunicación persuasiva, Arco

Del Barrio, J.A. y Borragán, A. (2011). Cómo atraer la atención hablando. Un reto para la enseñanza. Bor- dón. Revista de Pedagogía, 63(2), 15-26.

Del Giacco, L., Anguera, M.T. y Salcuni, S. (2020). The Action of Verbal and Non-verbal Communication in the Therapeutic Alliance Construction: A Mixed Methods Approach to Assess the Initial Interactions With Depressed Patients. Frontiers in Psychology, 11. Article number 234. https://doi.org/10.3389/ fpsyg.2020.00234

Demir-Lira, Ö.E., Asaridou, S.S., Raja Beharelle, A., Holt, A.E., Goldin-Meadow, S. y Small, S.L. (2018). Functional neuroanatomy of gesture-speech integration in children varies with individual differences in gesture processing. Developmental Science, 21(5). https://doi.org/1055/s-0033-1353447

Denervaud, S., Mumenthaler, C., Gentaz, E. y Sander, D. (2020). Emotion Recognition Development: Preliminary evidence for an effect of pedagogical practices. Learning and Instruction, 69, Article number 101353. j.learninstruc. 2020.101353 https://doi.org/10.1016/

Deutsch, F. (1947). Analysis of postural behavior. The Psychoanalytic Quarterly, 16(2), 195-213.

Diez, S. y Freijeiro, S. D. (2005). Técnicas de comunicación: la comunicación en la empresa. España: Ideaspropias Editorial.

D' Mello, S., Picard, R.W. y Graesser, A. (2007). Toward an affect-sensitive AutoTutor. IEEE Intelligent Systems, 22(4), 53-61.

Domínguez, E. (2009). La construcción de la imagen: Signos, comunicación y contexto en el diseño y las ciencias sociales. Colombia: UPB.

Dupertuis, V. y Moro, C. (2016). Self-directed ostensions and mediations of the adult at the age of 8-, 12-and 16 months. Integrative Psychological and Behavioral Science, 50(4), 621-633. https://doi.org/10.1007/ s12124-016-9350-x

Durand, K., Gallay, M., Seigneuric, A., Robichon, F. y Baudouin, J.Y. (2007). The development of facial emotion recognition: The role of configural information. Journal of Experimental Child Psychology, 97(1), 14-27. https://doi.org/10.1016/ j.jecp.2006.12.001

Durand, M.F., Martínez, M.S., Gago-Galvagno, L.G. y Elgier, A.M. (2020). El desarrollo temprano de la comunicación verbal y preverbal: Estudiando la importancia del juego. Revista Iberoamericana de Psicología, 13(1), 23-32.

Eco, U. y Volli, U. (1970). Introduzione a paralinguistica e 
cinesia. Milán: Bompiani

Efron, D. (1941). Gesture and environment. New York: King'S Crown Press.

Ekman, P. y Friesen, W.V. (1969). The repertoire of nonverbal behavior: Categories, origins, usage, and coding. Semiotica 1(1), 49-98.

Ekman, P. y Friesen, W.V. (1972). Hand movements. Journal of Communication, 22(4), 353-374. https:// doi.org/10.1111/j.1460-2466.1972.tb00163.x

Esteve-Gibert, N., Igualada, A. y Prieto, P. (2019). El gest com a facilitador i precursor del desenvolupament del llenguatge. Llengua, Societat i Comunicació, 17, 26-41.

Etchevers, G. N. (2006). Los nuevos códigos de la comunicación emocional utilizados en Internet. Revista Electrónica Teoría de la Educación, 7(2), 92-106.

Extremera, N., Mérida, S. y Sánchez, M. (2019). La importancia de la inteligencia emocional del profesorado en la misión educativa: impacto en el aula y recomendaciones de buenas prácticas para su entrenamiento. Voces de la Educación, Número especial 2, 74-97.

Flick, U. (2015). El diseño de investigación cualitativa. Madrid: Morata

Fong, F.T., Mondloch, C.J. y Nelson, N.L. (2020). Interactive situations reveal more about children's emotional knowledge. Journal of Experimental Child Psychology, $\quad 198 . \quad$ https://doi.org/10.1016/ j.jecp.2020.104879

Fossa, P. (2019). Lo representacional y lo expresivo: Dos funciones del lenguaje interior. Psicología Teoría e Pesquisa, 35, Artículo e365316. https:// doi.org/10.1590/0102.3772e35316

Gallo, L.E. (2009). El cuerpo en la educación da qué pensar: perspectivas hacia una educación corporal. Estudios Pedagógicos, 35(2), 232-242.

Gallo, L.E. (2017). Una didáctica performativa para educar (desde) el cuerpo. Revista Brasileira de Ciências do Esporte, 39(2), 199-205.

García, S.E., y Tully, E.C. (2020). Children's recognition of happy, sad, and angry facial expressions across emotive intensities. Journal of Experimental Child Psychology, 197. https://doi.org/10.1016/ j.jecp. 2020.104881

Gil-Olarte, M., Palomera, R. y Brackett, M.A. (2006). Relating emotional intelligence to social competence and academic achievement in high school students.
Psicothema, 18(Suppl.), 118-123.

Goleman, D. (2012). Inteligencia Emocional. Barcelona: Kairós.

Goldin-Meadow, S. (2005). Hearing gesture: How our hands help us think. Cambridge: Harvard University Press.

Gordillo, F., López, R.M., Mestas, L. y Corbi, B. (2014). Comunicación no verbal en la negociación: La importancia de saber expresar lo que se dice. Revista Electrónica de Psicología Iztacala, 17(2), 646-666.

Gordillo, F., Pérez, M.Á., Mestas, L., Salvador, J., Arana, J.M. y López, R.M. (2015). Diferencias en el Reconocimiento de las Emociones en Niños de 6 a 11 Años. Acta de Investigación Psicológica, 5(1), 1846 $-1859$. 30005-3

Gutiérrez-Torres, A. y Buitrago-Velandia, S. (2019). Las Habilidades Socioemocionales de los Docentes, herramientas de paz en la escuela. Praxis \& Saber, 10(24), 167-192. https:// doi.org/10.19053/22160159.v10.n25.2019.9819

Hargreaves, A. (2000). Mixed emotions: Teachers' perceptions of their interactions with students. Teaching and Teacher Education, 16(8), 811-826.

Herrera, L., Buitrago, R.E. y Avila, A.K. (2016). Empathy in future teachers of the Pedagogical and Technological University of Colombia. New Approaches in Educational Research, 5(1), 30-37. https:// doi.org/10.7821/naer.2016.1.136

Herrera, L., Buitrago, R.E. y Cepero, S. (2017). Emotional intelligence in Colombian primary school children. Location and gender. Universitas Psychologica, 16 (3), 165-174. http://dx.doi.org/10.11144/ javeriana.upsy16-3.eips

Herrera, L., Buitrago, R.E., Lorenzo, O. y Perandones, T.M. (2015). Psicología Positiva e Inteligencia Emocional en Educación. DEDiCA, Revista de Educação e Humanidades, 8, 139-153.

Holm, M. y Cekaite, A. (2021). Emotion socialization compassion or non-engagement - in young children's responses to peer distress. Learning Culture and Social Interaction, 28, Article number 100464. https://doi.org/10.1016/j.lcsi.2020.100462

Hwang, H.C. y Matsumoto, D. (2016). Facial expressions. In D. Matsumoto, H.C. Hwang, y M.G. Frank (Eds.), APA handbooks in psychology. APA handbook of nonverbal communication (pp. 257-287). Washington D.C.: American Psychological Associa- 
tion. https://doi.org/10.1037/14669-010

Igualada, A., Bosch, L. y Prieto, P. (2015). Language development at 18 months is related to multimodal communicative strategies at 12 months. Infant Behavior and Development, 39, 42-52. http:// doi.org/10.1016/j.infbeh.2015.02.004

Ip, K., Miller, A., Karasawa, M., Hirabayashi, H., Kazama, M., Wang, L., Kessler, D. y Tardif, T. (2021). Emotion expresión and regulation in three cultures: Chinese, Japanese and American preschoolers' reactions to disappontment. Journal of Experimental Child Psychology, 201, Article number 104972. https://doi.org/10.1016/j.jecp.2020.104972

Johnson, R.B. y Onwuegbuzie, A.J. (2004). Mixed methods research: A research paradigm whose time has come. Educational researcher, 33(7), 14-26.

Kachel, G., Hardecker, D. y Bohn, M. (2021). Young children's developing ability to integrate gestural and emotional cues. Journal of Experimental Child Psychology, 201, Article number 104984. https:// doi.org/10.1016/j.jecp.2020.104984

Kendon, A. (2004). Gesture: Visible action as utterance. Cambridge: Cambridge University Press.

Kleinginna, P.R. y Kleinginna, A.M. (1981). A categorized list of emotion definitions, with suggestions for a consensual definition. Motivation and Emotion, 5 (4), 345-379.

Knapp, M.L. y Hall, J.A. (2009). Nonverbal Communication in Human Interaction ( $7^{\mathrm{a}}$ ed.). México: Cengage Learning.

Koch, A. y Pollatos, O. (2015). Reduced facial emotion recognition in overweight and obese children. Journal of Psychosomatic Research, 79(6), 635-639. https://doi.org/10.1016/j.jpsychores.2015.06.005

Lapierre, A. y Aucouturier, B. (1977). Simbología del movimiento: Psicomotricidad y Educación. Barcelona (España): Editorial Científico Médica.

Lavelli, M. y Majorano, M. (2016). Spontaneous gesture production and lexical abilities in children with specific language impairment in a naming task. Journal of Speech, Language, and Hearing Research, 59(4), 784-796. https://doi.org/10.1044/2016_JSLHR-L-14 $-0356$

Lavelli, M., Barachetti, C. y Florit, E. (2015). Gesture and speech during shared book reading with preschoolers with specific language impairment. Journal of Child Language, 42(6), 1191. https://doi.org/ $10.1017 / \mathrm{S} 0305000914000762$
Leite, J. y Aureliano, T. (2016). O gesto apontar como catalizador nas cenas de atenção conjunta nas interações mãe-bebê. DLCV- João Pessoa, 10(2), 121125.

Linden, D. (2010). El cerebro accidental, la evolución de la mente y el origen de los sentimientos. Barcelona: Paidós.

Liszkowski, U. (2008). Before L1: A differentiated perspective on infant gestures. Gesture, 8(2), 180-196. https://doi.org/ 10.1075/gest.8.2.04lis

Lockwood, G., Dingemanse, M. y Hagoort, P. (2016). Sound-Symbolism Boosts Novel Word Learning. Journal of Experimental Psychology: Learning, Memory, and Cognition, 42(8), 1274-1281. http:// doi.org/10.1037/a0028642

Lüke, C. y Ritterfeld, U. (2014). The influence of iconic and arbitrary gestures on novel word learning in children with and without SLI. Gesture, 14(2), 204225. https://doi.org/10.1075/gest.14.2.04luk

Lüke, C., Ritterfeld, U., Grimminger, A., Liszkowski, U. y Rohlfing, K.J. (2017). Development of pointing gestures in children with typical and delayed language acquisition. Journal of Speech, Language, and Hearing Research, 60(11), 3185-3197. https:// doi.org/10.1044/2017_JSLHR-L-16-0129

Lüke, C., Ritterfeld, U., Grimminger, A., Rohlfing, K.J. y Liszkowski, U. (2020). Integrated Communication System: Gesture and Language Acquisition in Typically Developing Children and Children With LD and DLD. Frontiers in Psychology, 11, 118. https:// doi.org/10.3389/fpsyg.2020.00118

Mainela-Arnold, E. y Evans, J.L. (2014). Do statistical segmentation abilities predict lexical-phonological and lexical-semantic abilities in children with and without SLI?. Journal of Child Language, 41(2), 327-351. S0305000912000736 https://doi.org/10.1017/

Manwaring, S.S., Mead, D.L., Swineford, L. y Thurm, A. (2017). Modelling gesture use and early language development in autism spectrum disorder. International Journal of Language and Communication Disorders, 52(5), 637-651. https:// doi.org/10.1111/1460-6984.12308

Maricchiolo, F., Gnisci, A., Bonaiuto, M. y Ficca, G. (2009). Effects of different types of hand gestures in persuasive speech on receivers' evaluations. Language and Cognitive Processes, 24, 239-266. https://doi.org/10.1080/01690960802159929

Martí, E. (2017). Del cuerpo a la cultura: la necesidad de 
una mirada desde la psicología del desarrollo. Un comentario sobre el artículo Aprender más allá del cuerpo de Juan-Ignacio Pozo. Infancia y Aprendizaje, 40(4), 661-675. https:// doi.org/10.1080/02103702.2017.1364060

Matsumoto, D., Frank, M.G. y Hwang, H.S. (2012). Nonverbal communication: Science and applications. Londres: Sage

Mayer, J.D. y Salovey P. (1993). The intelligence of emotional intelligence. Intelligence, 17, 433-442. DOI: 10.1016/0160-2896(93)90010-3

Mayer, J.D. y Salovey P. (1997). What is emotional intelligence? En P. Salovey, y D. Sluyter (Eds.), Emotional development and emotional intelligence: implications for educators (pp. 3-34). New York: Basic Books.

Mayer, J.D. y Salovey P. (2009). ¿Qué es inteligencia emocional? En J. M. Mestre, y P. Fernández-Berrocal (Coords.), Manual de inteligencia emocional (pp. 25 -45). Madrid: Ediciones Pirámide.

Mayer, J.D., Caruso, D.R. y Salovey, P. (2016). The ability model of emotional intelligence: Principles and updates. Emotion Review, 8, 290-300. https:// doi.org/0.1177/1754073916639667

McNeill, D. (2016). Why we gesture: The surprising role of hand movements in communication. Cambridge: Cambridge University Press.

Meeren, H.K., Van Heijnsbergen, C.C. y De Gelder, B. (2005). Rapid perceptual integration of facial expression and emotional body language. Proceedings of the National Academy of Sciences, 102(45), 16518-16523.

Minto, A., Alva, E.A. y Arias, N. (2018). Los gestos deícticos y su impacto en el lenguaje productivo de infantes. Investigación y Práctica en Psicología del Desarrollo, 4, 1-17.

Moffitt, T.E., Arseneault, L., Belsky, D., Dickson, N., Hancox, R.J., Harrington, H., Houts, R., Poulton, R., Roberts, B.W., Ross, S., Sears, M.R., Thomson, W.M. y Caspi, A. (2011). A gradient of childhood self-control predicts health, wealth, and public safety. Proceedings of the National Academy of Sciences, 108(7), 2693-2698.

Mondloch, J.E., Bayram, E. y Finke, R.G. (2012). A review of the kinetics and mechanisms of formation of supported-nanoparticle heterogeneous catalysts. Journal of Molecular Catalysis A: Chemical, 355, 1-38.

Moreno, B. y Gálvez, M. (2010). La psicología positiva va a la escuela. Típica Boletín Electrónico de Salud Escolar, 1(6), 210-220.

Moreno-Nunez, A., Rodriguez, C. y Miranda-Zapata, E. (2020). Getting away from the point: the emergence of ostensive gestures and their functions. Journal of Child Language, 47(3), 556-578. https:// doi.org/10.1017/S0305000919000606

Mumford, K.H. y Kita, S. (2014). Children use gesture to interpret novel verb meanings. Child Development, 85(3), 1181-1189. https://doi.org/10.1111/ cdev. 12188

Murillo, E., Galera, N. y Casla, M. (2015). Gesture and speech combinations beyond two-word stage in an experimental task. Language, Cognition and Neuroscience, 30(10), 1291-1305. http:// doi.org/10.1080/23273798.2015.1066509

Nobe, S. (2000). Where do most spontaneous representational gestures actually occur with respect to speech. En D. McNeill (Ed.), Language and gesture (pp. 186-196). Cambridge: Cambridge University Press.

Ochsner, K.N., Ray, R.D., Cooper, J.C., Robertson, E.R., Chopra, S., Gabrieli, J.D. y Gross, J.J. (2004). For better or for worse: neural systems supporting the cognitive down- and upregulation of negative emotion. NeuroImage, 23, 483-499. https:// doi.org/10.1016/j.neuroimage.2004.06.030

Olderbak, S., Wilhelm, O., Hildebrandt, A. y Quoidbach J. (2019). Sex differences in facial emotion perception ability across the lifespan. Cognition and Emotion, 33(3) 579-588. https:// doi.org/10.1080/02699931.2018.1454403

Özçalışkan, Ş., Adamson, L.B. y Dimitrova, N. (2016). Early deictic but not other gestures predict later vocabulary in both typical development and autism. Autism, 20(6), 754-763. https://doi.org/ $10.1177 / 1362361315605921$

Palomera, R. (2017). Psicología Positiva en la Escuela: Un Cambio con Raíces Profundas. Papeles del Psicólogo, 38(1), 66-71.

Palomera, R., Briones, E. y Gómez-Linares, A. (2019). Formación en valores y competencias socioemocionales para docentes tras una década de innovación. Praxis y Saber, 10(24), 93-117. https:// doi.org/10.19053/22160159.v10.n25.2019.9116

Palomera, R., Briones, E., Gómez-Linares, A. y Vera, J. (2017). Cubriendo el vacío: mejorando las competencias sociales y emocionales del profesorado en formación. Revista de Psicodidáctica, 22(2), 142149. https://doi.org/10.1016/j.psicod.2017.05.002 
Pena, M., Extremera, N. y Rey, L. (2016). Las competencias emocionales: material escolar indispensable en la mochila de la vida. Padres y Maestros, 368, 6-10. https://doi.org/10.14422/pym.i368.y2016.001

Perlovsky, L. y Schoeller, F. (2019). Unconscious emotions of human learning. Physics of Life Reviews, 31, 257262. https://doi.org/10.1016/j.plrev.2019.10.007

Pert, C.B. (1986) La sabiduría de los receptores: neuropéptidos, emociones y cuerpo-mente. Advances Institute for the Advancement of Health, 3(3), 1-12.

Poyatos, F. (1994). La comunicación no verbal (Vol. 13). Madrid: Ediciones AKAL.

Pouw, W., Harrison, S.J. y Dixon, J.A. (2020). Gesturespeech physics: The biomechanical basis for the emergence of gesture-speech synchrony. Journal of Experimental Psychology: General, 149(2), 391. http://dx.doi.org/10.1037/xge0000646

Pozo, J.I. (2017) Más allá de la mente dual: la reconstrucción cultural de las representaciones encarnadas. Respuesta a los comentarios al artículo Aprender más allá del cuerpo. Infancia y Aprendizaje, 40(4), 713-741.

https:// doi.org/10.1080/02103702.2017.1306942

Quiñones, J.A. y Romero, J.E. (2020). Exploraciones sobre las concepciones y prácticas del cuerpo y la comunicación no verbal: la escuela, el proceso de aprendizaje y el aula de lengua extranjera. Revista Educación, 44(2), 430-449. http://dx.doi.org/10.15517/ revedu.v44i2.38945

Ramallo, F. (2019). La (re) invención del pasado como gesto de (des) composición de la Pedagogía. Sophia, colección de Filosofía de la Educación, (27), $217-$ 236. http://dx.doi.org/10.17163/soph.n27.2019.07

Repacholi, B.M. y Meltzoff, A.N. (2007). Emotional eavesdropping: Infants selectively respond to indirect emotional signals. Child Development, 78(2), 503521. https://doi.org/10.1111/j.14678624.2007.01012.x

Ricci y Cortesi (1980). Comportamiento no verbal. Paralenguaje, Kinésica e Interacción. Ediciones Itsmo.

Rodríguez, C., Moreno-Núñez, A., Basilio, M. y Sosa, N. (2015). Ostensive gestures come first: their role in the beginning of shared reference. Cognitive Development, 36, 142-149. https://doi.org/10.1016/ j.cogdev.2015.09.005

Rulicki, S. y Cherny, M. (2012). CNV comunicación noverbal: cómo la inteligencia emocional se expresa a través de los gestos. Buenos Aires: Ediciones Grani- ca SA.

Salo, V.C., Ferrari, P.F. y Fox, N.A. (2019). The role of the motor system in action understanding and communication: Evidence from human infants and nonhuman primates. Developmental Psychobiology, 61 (3), 390-401. https://doi.org/ 10.1111/infa.12229

Salgado, D.P. y Otero, M.R. (2020). Enseñanza por investigación en un curso de matemática de nivel universitario: los gestos didácticos esenciales. Revista do Programa de Estudos Pós-Graduados em Educação Matemática, 22(1). http://dx.doi.org/10.23925/19833156.2020v22i1p532-557

Salisch, M., Haenel, M. y Freund, P.A. (2013). Emotion understanding and cognitive abilities in young children. Learning and Individual Differences, 26, 1519. https://doi.org/10.1016/j.lindif.2013.04.001

Salzberger-Wittenberg, I. y Osborne, E. (1983). The emotional experience of learning and teaching. Londres: Routledge and Kegan Paul.

Sánchez, R. (2019). Política de gestos. La aristocracia contra la monarquía democrática de Amadeo de Saboya. Pasado y Memoria. Revista de Historia Contemporánea, 18, 19-38. https://doi.org/10.14198/ PASADO2019.18.02

Santiago, D.T., Mateo, C.G., Guijarro, S.T., Fernández, L.D. y Castro, J.L.A. (2019). Estudio de bases de datos para el reconocimiento automático de lenguas de signos. Hesperia: Anuario de Filología Hispánica, 22, 145-160.

Sekine, K., Sowden, H. y Kita, S. (2015). The development of the ability to semantically integrate information in speech and iconic gesture in comprehension. Cognitive Science, 39(8), 1855-1880. https://doi.org/ $10.1111 /$ cogs. 12221

Shablico, S. (2012). La comunicación no verbal en el aula, un análisis en la enseñanza disciplinar. Cuadernos de Investigación Educativa, 3(18), 99-121.

Shapiro, L. (2008). La inteligencia emocional de los niños. Una guía para padres y maestros. Zeta.

Singer, M.A. y Goldin-Meadow, S. (2005). Children learn when their teacher's gestures and speech differ. Psychological Science, 16(2), 85-89.

Tartas, V. (2017). Hacia un enfoque integrador de cuerpo, mente y cultura: nuevos desafíos y perspectivas para la psicología educativa y del desarrollo. Un comentario sobre el artículo Aprender más allá del cuerpo de Juan-Ignacio Pozo. Infancia y Aprendizaje, 40
(4),
676-688
https:// 
doi.org/10.1080/02103702.2017.1364061

Tur-Porres, G. y Ires-Correa, W. (2020). Educating With and From the Body: Culture, Body and Body Reeducation. Revista Electrónica Educare, 24(2), 220239. http://doi.org/10.15359/ree.24-2.11

Usler, E., Foti, D. y Weber, C. (2020). Emotional reactivity and regulation in 5-to 8-year-old children. En ERP study of own-age face processing. International Journal of Psychophysiology, 156, 60-68. https:// doi.org/10.1016/j.ijpsycho.2020.07.004

Van den Stock, J., Righart, R. y De Gelder, B. (2007). Body expressions influence recognition of emotions in the face and voice. Emotion, 7(3), 487-494. https://doi.org/10.1037/1528-3542.7.3.487

Vogt, S. y Kauschke, C. (2017). Observing iconic gestures enhances word learning in typically developing children and children with specific language impairment. Journal of child language, 44(6), 1458-1484. https://doi.org/10.1017/S0305000916000647

Walle, E.A., Reschke, P.J., Camras, L.A. y Campos, J.J. (2017). Infant differential behavioral responding to discrete emotions. Emotion, 17(7), 1078. https:// dx.doi.org/ 10.1037/emo0000307

Waters, G.M. y Beck, S.R. (2015). Verbal Information Hinders Young Children's Ability to Gain Modality Specific Knowledge. Infant and Child Development, 24(5), 538-548. https://doi.org/10.1002/icd.1901

Weick, K.E. (1968). Systematic observational methods. In G. Lindzey y E. Aronson (Eds.), Handbook of Social Psychology (pp. 357-451). New York: AddisonWesley.

Weismer, S.E. y Hesketh, L.J. (1993). The influence of prosodic and gestural cues on novel word acquisition by children with specific language impairment. Journal of Speech, Language, and Hearing Research, 36(5), 1013-1025. https://doi.org/10.1044/ jshr.3605.1013

Widen, S.C. y Russell, J.A. (2003). A closer look at preschoolers' freely produced labels for facial expressions. Developmental Psychology, 39(1), 114-128. https://doi.org/10.1037/0012-1649.39.1.114

Yadav, K.S. y Singha, J. (2020). Facial expression recognition using modified Viola-John's algorithm and KNN classifier. Multimedia Tools and Applications, 79, 13089-13107 https://doi.org/10.1007/s11042019-08443-x 